\title{
Stoichiometry changes by selective vacancy formation on (110) surfaces of III-V semiconductors: Influence of electronic effects
}

\author{
U. Semmler, M. Simon, Ph. Ebert, ${ }^{\text {a) }}$ and K. Urban \\ Institut für Festkörperforschung, Forschungszentrum Jülich GmbH, 52425 Jülich, Germany
}

(Received 11 September 2000; accepted 4 October 2000)

\begin{abstract}
We investigated the kinetics of thermal formation of anion vacancies and the subsequent stoichiometry changes on (110) cleavage surfaces of III-V semiconductors by scanning tunneling microscopy. We found that the rate of spontaneous formation of monovacancies depends very sensitively on the doping of the underlying semiconductor and the concentration of surface vacancies. It is shown that the position of the Fermi energy at the surface is the major electronic influence on the energy barrier height for the vacancy formation. We found barrier heights in the range of 1.1-1.3 eV for GaAs and InP. The physical factors affecting the vacancy formation and the surface stoichiometry are discussed in detail. (C) 2001 American Institute of Physics.
\end{abstract}

[DOI: $10.1063 / 1.1328412$ ]

\section{INTRODUCTION}

It is well known that point defects not only affect the electronic properties of compound semiconductors if they occur in sufficient concentrations but, in addition, a selective vacancy formation can modify the semiconductors' stoichiometry. It is, however, rather difficult to know or predict under which condition(s) a particular type of defect occurs in concentrations relevant for the macroscopic properties of the compound semiconductor material. In order to be able to predict defect concentrations one has to determine all physical and chemical factors influencing the formation of these defects. So far, however, most works addressed only the case of thermal equilibrium, where the concentrations of defects are controlled by their formation energies. Real equilibrium conditions exist, however, only in few exceptional cases. Most semiconductor devices are rather grown under nonequilibrium conditions to obtain optimal properties. Thus for most technological interesting systems it is crucial to understand the kinetics of defect formation and in particular the physical and chemical effects influencing the key parameters of defect formation, such as the energy barrier. Such knowledge may lead to a detailed understanding of the defect formation processes in semiconductors and the possibility to predict theoretically the macroscopic properties of semiconductors on the basis of defect concentrations.

The investigation of the formation kinetics of point defects in the bulk is, however, rather difficult, because it is necessary to identify the types and concentrations of defects present, something which is rarely achieved for bulk point defects. ${ }^{1,2}$ Therefore, we turn to surface defects on III-V semiconductor surfaces as model system. In contrast to bulk defects, surface defects can be directly imaged by scanning tunneling microscopy ${ }^{3}$ and thus their kinetics of formation are easily accessible. ${ }^{4}$

In this paper we investigate the factors influencing the kinetics of thermal formation of anion vacancies on (110)

${ }^{\text {a)} E l e c t r o n i c ~ m a i l: ~ p . e b e r t @ f z-j u e l i c h . d e ~}$ cleavage surfaces of different III-V semiconductors by scanning tunneling microscopy. We demonstrate that rate of spontaneous formation of monovacancies depends very sensitively on the doping of the underlying semiconductor and on the concentration of surface vacancies. It is shown that the position of the Fermi energy at the surface is the major electronic influence on the energy barrier height for the vacancy formation. We also discuss the reciprocal influence of vacancies and the Fermi energy at the surface.

\section{EXPERIMENT}

We investigated the vacancy formation for a large set of differently $p$-doped GaAs and InP single crystals. All GaAs crystals were doped with $\mathrm{Zn}$, whereas most InP crystals were doped with $\mathrm{Cd}$. Only the highest doped InP crystal had Zn dopants. The carrier concentration of the GaAs crystals were $1.8 \times 10^{17}, 2.5 \times 10^{18}$, and $1.1 \times 10^{19} \mathrm{~cm}^{-3}$. The different InP crystals had carrier concentrations of $5 \times 10^{17}, 1.1 \times 10^{18}$, and $8 \times 10^{18} \mathrm{~cm}^{-3}$. In order to produce clean and defect free surfaces we cleaved the crystals in ultrahigh vacuum along one of their (110) planes. The investigations of the cleavage surface by scanning tunneling microscopy (STM) started immediately after cleavage. All measurements were made at room temperature to ensure a high stability and an exact measurement of the temperature. Furthermore at higher temperatures additional effects, such as vacancy diffusion into the bulk may affect the measured surface vacancy formation rates too much. From the STM images we deduced directly the rate of formation of vacancies on the surface and the rate of composition changes as a function of the carrier concentration, material, vacancy concentration, and time after cleavage.

\section{RESULTS}

Directly after cleavage the (110) surfaces of all III-V semiconductors are essentially free of defects with the exception of a few cleavage induced defects. Thus the surfaces 

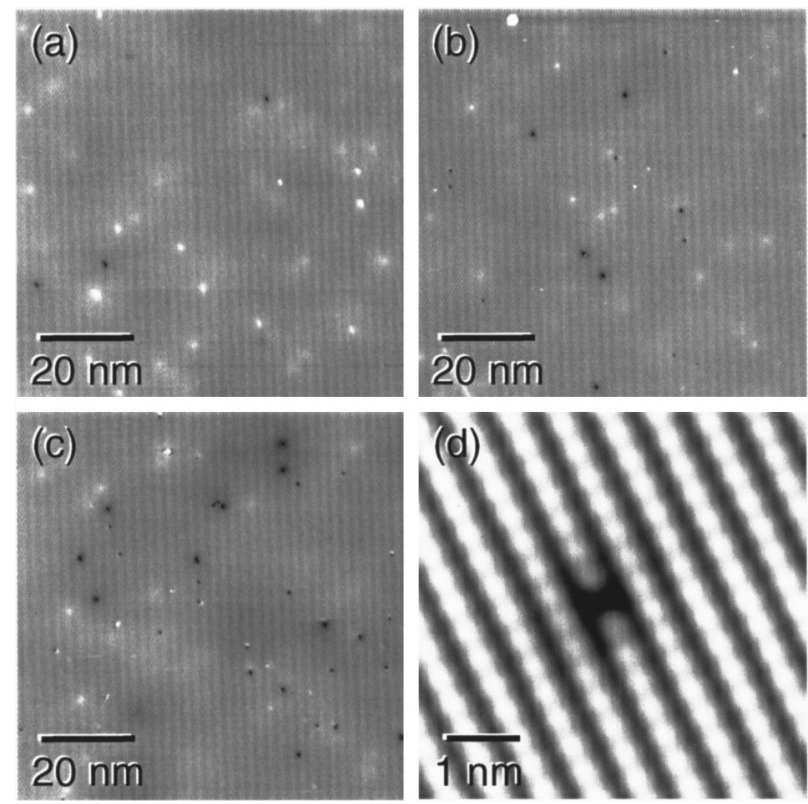

FIG. 1. Constant-current STM images of the occupied states of the cleaved GaAs(110) surface (a) 2.6, (b) 93.5, and (c) $381.5 \mathrm{~h}$ after cleavage. The dark contrast features are positively charged As monovacancies, whose concentration increases with time. Frame (d) shows a high resolution STM image of the occupied density of states acquired at $-2.6 \mathrm{~V}$. Clearly one occupied dangling bond in the surface is missing. This is due to the missing As atom at the As vacancy site. The carrier concentration of the sample has been $2.5 \times 10^{18} \mathrm{~cm}^{-3}$.

consist directly after cleavage of an equal number of anions (group V atoms) and cations (group III atoms). All other features on the surfaces are bright contrasts, whose concentrations scale with the dopant concentration. From this we conclude that the bright contrasts are individual electrically active dopant atoms in agreement with previous works. ${ }^{5-9}$ With increasing time after cleavage we found in addition to the bright contrast dark contrast features as shown in Figs. 1(a)-1(c) for GaAs. These dark contrast features indicate the presence of a localized positive charge at the defects. ${ }^{10-12}$ Figure 1(d) shows a high resolution image of the occupied density of states of the defect. The defects consist of one missing occupied dangling bond in the occupied state images and no missing empty dangling bond. This structure has been previously identified to be that of anion vacancies on (110) surfaces of III-V semiconductors. ${ }^{3,13-19}$ The vacancies were also shown to have a $+1 e$ charge on $p$-doped surfaces. ${ }^{9,20,21}$

With increasing time after cleavage we observed that the concentration of the As vacancies increased strongly [compare Figs. 1(a)-1(c)] on each of the GaAs samples investigated in agreement with previous reports. ${ }^{4,22,23}$ We also observed that the vacancy concentration at a particular time after cleavage increases for samples with higher carrier concentrations (Fig. 2). Note that we found the same behavior for $\mathrm{GaAs}$ as for $\operatorname{InP}(110)$ (Ref. 4) cleavage surfaces. On $p$-doped $\mathrm{GaP}(110)$ surfaces we also observed $\mathrm{P}$ vacancies on the surface after some time, but their concentration was rather low, presumably due to a low carrier concentration of the GaP sample [ $\mathrm{Zn}:(1.7-5.8) \times 10^{17} \mathrm{~cm}^{-3}$ ], as discussed below.

Figure 3(a) shows the quantitative concentration values
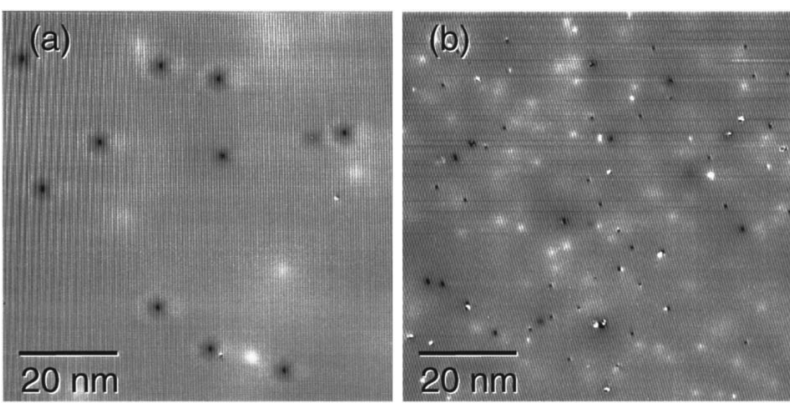

FIG. 2. Constant-current STM images of the occupied states of (110) cleavage surfaces of $\mathrm{Zn}$-doped GaAs with carrier concentrations of (a) 1.8 $\times 10^{17} \mathrm{~cm}^{-3}$ and (b) $1.1 \times 10^{19} \mathrm{~cm}^{-3}$. The images were obtained (a) 105 and (b) $99 \mathrm{~h}$ after cleavage. The STM images demonstrate that the vacancy concentration increases with increasing carrier concentration of the samples.

of As vacancies on the differently doped GaAs(110) surfaces as deduced from the STM images. We avoided observing tip-induced vacancies by never scanning a surface area twice and by acquiring the STM images with tunneling conditions
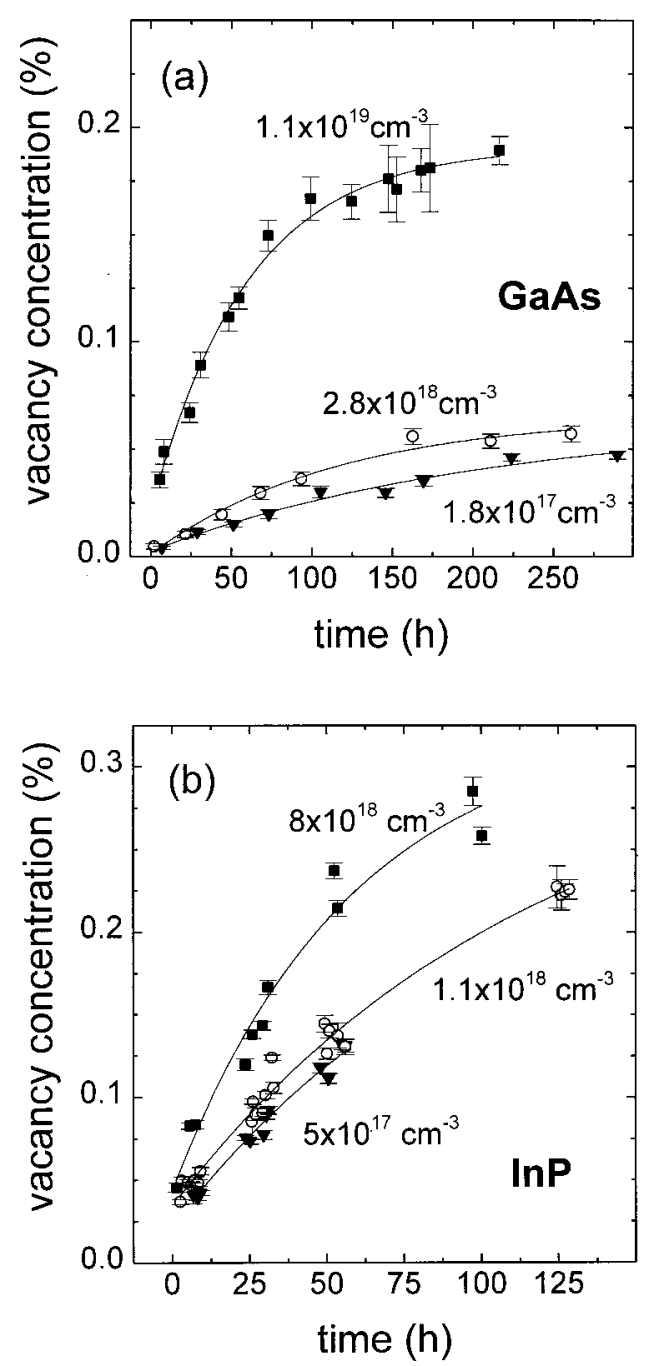

FIG. 3. Concentrations of anion vacancies in fraction of anion surface lattice sites as a function of time after cleavage at room temperature. (a) Shows the data sets for three $\mathrm{GaAs}(110)$ surfaces with different carrier concentrations. The solid lines should guide the eye. For comparison, (b) shows the similar data sets for $\operatorname{InP}(110)$ surfaces (Ref. 4). 
under which tip-induced migration and formation events were found to be negligible. ${ }^{3}$ Thus the observed increase of the vacancy concentration can only be due to a thermal formation of vacancies. Note that the scattering of the concentration values is due to local inhomogeneities of the dopant concentration $^{24}$ and not due to insufficient statistics. The quantitative data shows more clearly three trends. First, the vacancy concentration increases for each sample with time. Second, on each sample the vacancy formation rate is decreasing with time, such that a saturation is suggested. Third, the vacancy concentration at constant time increases with increasing carrier concentration. The vacancy formation is thus particularly strong for highly doped samples. This effect is even more pronounced in $\mathrm{Zn}$-diffused GaAs, where As vacancy concentrations of $1 \%$ or more of the As surface sites can be observed even at room temperature. Such a concentration can only be reached for lower doping concentrations after heat treatments of the surfaces. ${ }^{22}$ Note that the data in Fig. 3(a) is scaled such that it directly gives the stoichiometry changes toward the Ga-rich side. Thus the surface becomes spontaneously slightly Ga enriched or As denuded.

Figure 3(b) shows for comparison the quantitative P vacancy concentrations observed at room temperature on three differently doped $\operatorname{InP}(110)$ surfaces. ${ }^{4}$ The data exhibits the same three trends, i.e., the increase of the vacancy concentration for each sample with time, the decrease of the formation rate with time, and the increase of the concentration at constant time with increasing carrier concentration of the samples. In fact rather little differences can be observed for $\mathrm{GaAs}$ and InP except that the vacancy concentration is always somewhat larger for $\operatorname{InP}(110)$ surfaces. Again the surface is becoming In rich and $\mathrm{P}$ poor.

\section{DATA ANALYSIS AND DISCUSSION}

In order to analyze the data, we have to address what physical quantities govern the initial formation of the defects. In case of thermal equilibrium or in case of indefinite time after cleavage the vacancies observed on the surface can reach their equilibrium concentration. The equilibrium concentrations are controlled by the vacancy formation energy, which is the difference in energy between the initial state with no vacancy in the surface and final state with a vacancy in the surface (see Fig. 4). These quantities can be calculated theoretically, however, only very few absolute values were reported so far. ${ }^{25}$ The kinetic increase of the vacancy concentration is, however, governed by the energy barrier for the formation of the vacancy. Figure 4 shows schematically the energy along the reaction path. The maximum energy relative to the initial state (on the left) is the energy barrier, which has to be overcome for the formation of the vacancy. At present nothing is known about the details of the reaction path during which the bonds to the neighboring atoms have to be broken. However, this is also not necessary for the present data analysis. We rather provide data, which may help to clarify the unknown details.

At this stage we consider the different thermal processes, which can affect the concentration of surface vacancies. Three processes can occur. These are first, the thermal formation of vacancies increasing the vacancy concentration,

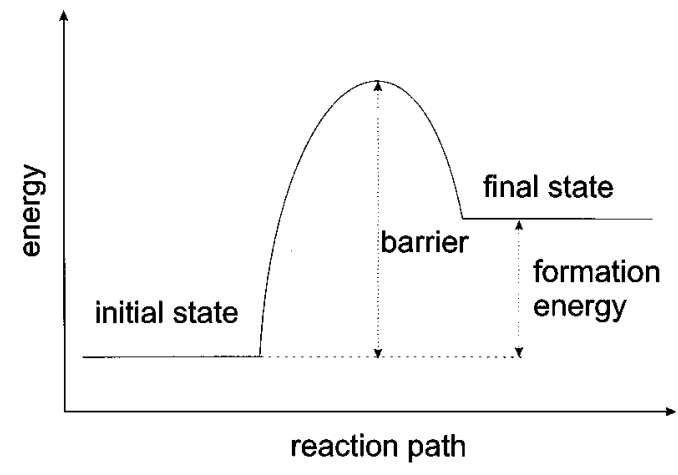

FIG. 4. Schematic drawing of the energy along the reaction path during the formation of a vacancy. On the left is the energy level of the defect-free surface (initial state), whereas the right-hand side is the energy of the surface after the formation of a vacancy (final state).

second, a possible diffusion of surface vacancies into the bulk crystal, and third, adatoms annihilating surface vacancies. The latter two processes reduce both the surface vacancy concentration. In the following we have to assess, which of the processes are relevant for the current experimental conditions.

A thermal diffusion of the surface vacancies into the bulk has been shown to occur above $140{ }^{\circ} \mathrm{C} .{ }^{9}$ With increasing temperature the in-diffusion plays an increasing role, however, at room temperature this effect is negligible, because on the one hand the low activation temperature and on the other hand the rather small vacancy concentrations at the surface. Furthermore the low vacancy concentration reduces the repulsive interactions between the positively charged vacancies and thus one of the key driving forces for indiffusion disappears. Below we will address the effect of Coulomb interactions in more detail.

Thus the increase of the concentration of vacancies per time interval $\mathrm{d} n_{V} / \mathrm{d} t$ is ${ }^{26}$

$$
\frac{\mathrm{d} n_{V}}{\mathrm{~d} t}=v \cdot n_{0} \cdot\left(1-\alpha n_{V}\right) \cdot e^{-(B / k T)}-K \cdot n_{V} \cdot n_{A}
$$

if we take the two remaining mechanisms into account. In the formula $v$ is the attempt frequency with which an atom attempts to leave its lattice site to form a vacancy, $B$ is the height of the energy barrier for the formation of the vacancy (see Fig. 4), $k$ is Boltzmann's constant, $n_{0}$ is the number of anion lattice sites per area in the surface layer, $K$ is the rate coefficient for the adatom-vacancy recombination process, and $n_{A}$ is the concentration of adatoms. The factor (1 $-\alpha n_{V}$ ) takes into account that the presence of a charged vacancy reduces effectively the concentration of lattice sites available for vacancy formation by $\alpha n_{V}$ due to a screened Coulomb repulsion between the vacancies ${ }^{21}(\alpha$ is the average surface area around each vacancy where no further vacancy can be formed). For an exact determination of the area $\alpha$ it would be necessary to know the details of the carrier concentration dependence of the screening and interaction potentials around a charged vacancy. Unfortunately, the interaction potential is only known so far for a single dopant concentration $^{21}$ and nothing is known about the carrier concentration dependence for surface screening (unlike bulk 
screening ${ }^{24,27}$ ). Therefore we have to restrict our analysis to vacancy concentrations where vacancy-vacancy interactions are negligible and $\alpha$ can be approximated by 1 unit cell size (because no second vacancy can be formed where a vacancy is present already). However, for low vacancy concentration as investigated here even this effect is negligible and the factor $\left(1-\alpha n_{V}\right)$ can indeed be approximated well enough by 1. We performed self-consistency tests showing that our data are well within this approximation for the vacancy concentrations investigated. Note that the screened Coulomb interaction between the vacancies limits the maximum reachable vacancy-induced nonstoichiometry of the surface.

The low concentration limit of the data analysis has also another advantage. It reduces the number of adatoms to such a degree that the adatom-vacancy recombination part can be neglected, too. Furthermore the adatoms were shown to be able to desorb thus reducing the probability of adatomvacancy recombination events even more. ${ }^{22}$ Thus within these limits the vacancy concentration is essentially representing the deviation of the surface stoichiometry.

With these low-vacancy concentration approximations, Eq. (1) can be simplified to

$$
\frac{\mathrm{d} n_{V}}{\mathrm{~d} t}=v \cdot n_{0} \cdot e^{-(B / k T)} .
$$

On the basis of this equation, it is possible to determine from the rate of vacancy formation $\mathrm{d} n_{V} / \mathrm{d} t$ the energy barrier $B$ for the vacancy formation if we know the attempt frequency $v$. The attempt frequency can be approximated by either the Debye frequency [GaAs: $7.6 \times 10^{12} \mathrm{~Hz}$ (Ref. 28); InP: 8.8 $\times 10^{12} \mathrm{~Hz}$ (Ref. 29)] or the phonon frequency. All typical phonon frequencies for GaAs and $\operatorname{InP}(110)$ surfaces are between $2 \times 10^{12}$ and $10 \times 10^{12} \mathrm{~Hz}^{30-37}$ For the current purpose we thus took a range of $1 \times 10^{12}-1 \times 10^{13} \mathrm{~Hz}$ for the attempt frequency for GaAs and InP. In the next step we extracted the rate of vacancy formation $\mathrm{d} n_{V} / \mathrm{d} t$ from the slope of the concentration data in Figs. 3(a) and 3(b) by fitting an exponential function to the measured data. Using the slope at infinitely small vacancy concentration $(t \rightarrow 0)$ we obtain for GaAs and InP surfaces a range for the value of the barrier height $B$ of $1.14-1.28 \mathrm{eV}$ and $1.14-1.23 \mathrm{eV}$, respectively. The width of the range of the values arises mostly from the uncertainty of the attempt frequency. We also included other effects, such as temperature, time, and statistical errors, but they do not affect the value for the barrier height that much.

At this stage we address first the absolute values of the barrier height. To our knowledge no values for the energy barrier height for the formation of a vacancy on a (110) surface of a compound semiconductor has been calculated so far. We thus can only check the consistency of our results with the theoretical calculations by comparing our barrier with the calculated formation energy. For this we have to recall that the barrier height is always larger than or equal to the formation energy. Calculations yielded formation energies of 1-1.8 eV for positively charged As vacancies on $p$-doped GaAs(110) and $0.4-1 \mathrm{eV}$ for the positively charged $\mathrm{P}$ vacancy on $p$-doped $\operatorname{InP}(110){ }^{25}$ The variations of the formation energies are due to the variation of the composition from anion to cation-rich materials. Most III-V semiconduc- tors are typically anion-rich in the bulk, however, we cannot determine to what degree. In addition, it is unclear how to define the chemical potential at the surface on which the vacancies are formed, since the surface is under Langmuir conditions and not in equilibrium Knudsen conditions. Within these limitations the calculated values can thus be considered to be consistent with our barrier heights. Diffusion barriers of vacancies on the GaAs(110) surface of 1.3 and $1.5 \mathrm{eV}$ also support the size of our barrier heights. ${ }^{38,39}$

A closer look at the values of the barrier height shows that the barrier height increases with increasing carrier concentration of the samples for GaAs as well as for $\operatorname{InP}(110)$ surfaces.

In order to extract only the carrier concentration dependence of the barrier height, we concentrated on the relative changes of the barrier as a function of the position of the Fermi energy. The discussion in terms of relative changes in barrier height has the advantage that the relative values are not affected by the attempt frequency and thus the largest source of uncertainty is removed. Furthermore the temperature measurement error is also reduced to the relative error. The restriction to relative changes allows us to probe significantly smaller changes of the barrier height.

The relative variations of the barrier height as a function of the position of the Fermi energy in the crystals are shown in Fig. 5 for GaAs (filled circles) and InP (filled triangles). The data points were extracted from the vacancy concentration values extrapolated to the cleavage time. Thus the Fermi level at the surface is given by that of the bulk crystal. ${ }^{3}$ For the calculation of the Fermi level position from the carrier concentrations we took the presence of degenerate carrier systems into account. It can be clearly seen that the barrier height increases with the Fermi energy shifting toward the center of the band gap. Thus for samples with a Fermi energy far above the valence band maximum it will be increasingly difficult to form vacancies. This is in agreement with the observation that on $n$-doped GaAs no As vacancies are formed. ${ }^{13}$

For negatively charged Ga vacancies on GaAs(110) surfaces the situation is reversed. On $n$-doped surfaces they are formed in rather high concentrations, ${ }^{2,13}$ while on $p$-doped surfaces Ga vacancies were not observed yet. ${ }^{13}$ Thus, the interpretation in terms of formation barrier heights yields that the energy barrier for the formation of negatively charged Ga vacancies will decrease from $p$ - to $n$-doped GaAs samples, which is the reversed trend compared to positively charged anion vacancies. This suggests that positively and negatively charged defects are formed spontaneously on $p$ and $n$-doped surfaces, respectively.

For a deeper understanding of the physical origins of the carrier concentration dependent barrier height, we turn again to Eq. (2). Equation (2) describes an apparently linear dependence of the vacancy concentration as a function of time, if the energy barrier $B$ is constant. A close look at the data in Fig. 3 shows, however, that this is indeed not the case. The curvature suggests that the barrier height increases apparently with time for each of the samples investigated. There may be several origins of this effect: (i) The curvature could be due to the approximation of no vacancy-vacancy interac- 


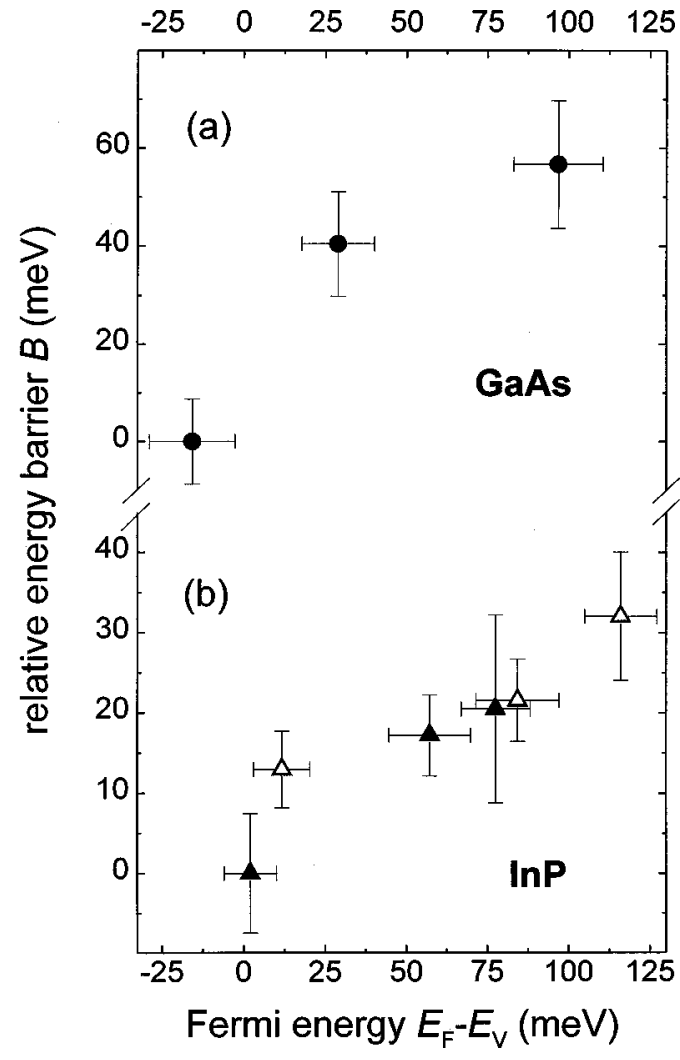

FIG. 5. Relative changes of the barrier height $B$ for the formation of positively charged anion vacancies on $p$-doped (110) surfaces of GaAs (a) and InP (b) as a function of the position of the Fermi energy on the surface. Filled symbols show the data for surface Fermi level positions equal to those in the bulk GaAs and InP (limit of zero vacancy concentration) and open triangles show data for Fermi level positions determined by the bulk and by the surface band bending induced by the presence of positively charged surface vacancies.

tions. (ii) The increasing concentration of vacancies introduces an increasing concentration of positive charges at the surfaces, which in turn induce a band bending at the surface, which shifts the Fermi level toward midgap at the surface. (iii) Due to the increased vacancy concentration the adatom concentration increased, too, and subsequently the adatomvacancy recombination probability is higher. (iv) The stoichiometry changes increase the effective formation barrier.

In order to discriminate between these different possibilities, we first focus on the surface band bending, because it is well known that under the higher charged vacancy concentrations a band bending develops. ${ }^{19}$ The magnitude of band bending can be calculated by taking into account that the charge per surface area in the surface layer $Q_{\mathrm{ss}}$ introduced by the charged anion vacancies is compensated by a charge density per surface area in a depletion layer $Q_{\mathrm{sc}}$ below the surface due to a redistribution of the free carriers. ${ }^{40}$ The charge per surface area for a concentration $n_{V}$ of $+1 e$ charged surface vacancies is

$$
Q_{\mathrm{ss}}=\frac{e \cdot n_{V}}{\exp \left(\left(E_{F}-E_{\mathrm{sd}}\right) / k T\right)+1},
$$

where $E_{F}$ is the Fermi energy. The difference in energy of the charge-transition level $E_{\mathrm{sd}}$ of the vacancy and the Fermi level $E_{\mathrm{sd}}-E_{F}$ is given by $\left(E_{\mathrm{sd}}-E_{\mathrm{sv}}\right)-\left(E_{v}-E_{\mathrm{sv}}\right)-\left(E_{F}\right.$ (a)

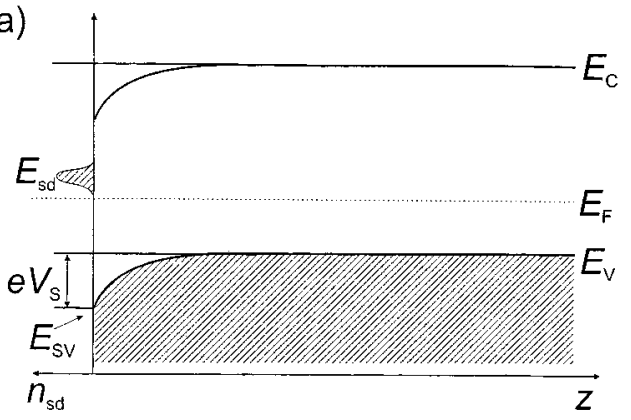

(b)

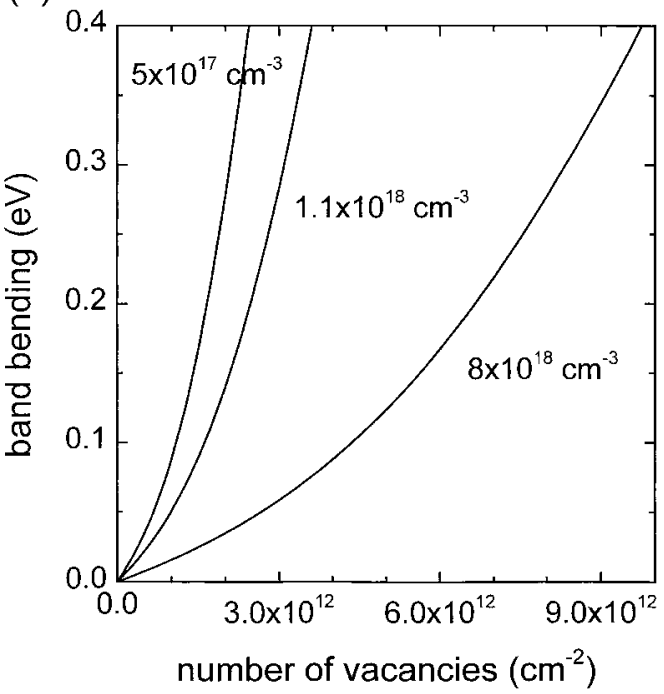

FIG. 6. (a) Schematic drawing of the band bending region at the surface induced by the presence of positive surface charges. (b) Calculated surface band bending as a function of the concentration of positively charged $\mathrm{P}$ vacancies on $p$-doped $\operatorname{InP}(110)$ surfaces for the carrier concentrations of the three investigated samples.

$\left.-E_{v}\right)$ [for the definition, see Fig. 6(a)], where $E_{v}-E_{\mathrm{sv}}$ is the band bending $e V_{s}$ measured by photoelectron spectroscopy at the surface $\left(E_{v}\right.$ and $E_{\mathrm{sv}}$ are the position of the valence band in the bulk and at the surface, respectively). The change density per surface area in the depletion layer $Q_{\text {sc }}$ compensating $Q_{\mathrm{ss}}$ is

$$
Q_{\mathrm{sc}}=\sqrt{2 \varepsilon_{r} \varepsilon_{0} \cdot n \cdot k T \cdot\left(\exp \left(-e V_{s} / k T\right)+\left(e V_{s} / k T\right)-1\right)}
$$

with $n$ being the concentration of charge carriers introduced by the dopant atoms. The energy difference between the Fermi energy and the valence band maximum in the bulk has been determined from the carrier concentration for each sample using the proper Fermi-Dirac integrals.

On this basis it is possible to calculate using Eqs. (3) and (4) the band bending as a function of the vacancy concentration [Fig. 6(b)]. For that it is necessary to use the energy of the $(+/ 0)$ charge-transition level of the vacancy relative to the valence band maximum $\left(E_{\mathrm{sd}}-E_{\mathrm{sv}}\right)$, which was found to be $(0.75 \pm 0.1) \mathrm{eV}$ at room temperature for the $\mathrm{P}$ vacancy on $\operatorname{InP}(110)$ surfaces. ${ }^{19}$ So far no values were experimentally measured for other vacancies. Thus we perform the analysis only for InP. Note that theoretical values for other 
material $^{17,18}$ deviate too much, mostly due to insufficiencies inherent in the calculational methods used, such that the calculated charge transition levels cannot be used for a reliable analysis of our data. Figure 6(b) shows the band bending calculated for the three InP samples for lower vacancy concentrations. This band bending has to be taken into account for the determination of the Fermi level for nonzero vacancy concentrations.

The open triangles in Fig. 5 show the barrier height for the $\mathrm{P}$ vacancy formation on $\operatorname{InP}(110)$ surfaces for each of the three InP samples at one specifically chosen nonzero vacancy concentration. We chose for the three data points (open triangles in Fig. 5) vacancy concentrations of about $0.1 \%-$ $0.15 \%$, because for these rather low vacancy concentrations the assumption of no vacancy-vacancy-interactions is still sufficiently fulfilled. In addition, although the vacancy concentrations are still rather low, they already induce a sufficiently large band bending at the surface shifting the band edges relative to the Fermi energy (this results in the shifted Fermi level for each of the three samples). The three data points which include a surface band bending agree very well with the data for infinitely small vacancy concentration where the Fermi level at the surface is governed by the Fermi level of the bulk semiconductors. All data points suggest a linear dependence of the barrier height for vacancy formation on the position of the Fermi energy on the surface. The excellent agreement of the two sets of data points with surface band bending and with no band bending at the surface shows that the barrier is only affected by the position of the Fermi energy at the surface and not by that in the bulk crystal.

Furthermore, the agreement also shows that at the vacancy concentrations studied here the approximation of no vacancy-vacancy interactions is sufficient. Only at higher vacancy concentrations we indeed found an increasingly significant modification of the effective barrier by vacancyvacancy interactions. In fact vacancy-vacancy interactions increase the apparent barrier considerably. It is also conceivable that at very high vacancy concentrations the stoichiometry changes will affect the vacancy formation via some mechanism not detectable at our low vacancy concentrations.

The possibility of an increased adatom concentration can also be ruled out, because with increasing adatom concentration the probability of adatom-adatom binding and subsequent desorption increases, too. This effect reduces the adatom concentration rather effectively, because we found rarely adatoms and in a previous high-temperature study neither adatoms were found in high concentrations. ${ }^{22}$ Thus at low vacancy concentrations the vacancy formation is governed by the position of the Fermi energy at the surface. This conclusion is further supported that the same data is obtained for $\mathrm{Cd}$ and $\mathrm{Zn}$ doped material.

At this stage we discuss possible origins of the dependence of the barrier height on the Fermi energy. First, one has to recall that in order to form a positively charged vacancy it is necessary to separate electrical charges. This charge separation has been suggested ${ }^{4}$ to occur simultaneously or shortly after the formation and relaxation of the vacancy. In such a case the charge separation may affect the barrier height. This influence on the barrier could arise from the fact that the formation energy of a positively charged defect increases linearly with the Fermi energy moving toward midgap, because the electron freed by the vacancy formation has to be moved above the Fermi energy. It is now conceivable that the final state can also affect to some degree the barrier height. In fact the observed increase of the barrier height with the Fermi energy moving toward midgap is consistent with the direction of change excepted for a positively charged defect.

Another possibility would be that the charge separation occurs at the time the vacancy formation process reaches the maximum barrier height along its reaction path. In such a case it is natural to assume that the barrier height will be directly influenced by the position of the Fermi energy. This possibility suggests that the observed dependence of the barrier height would be a signature of a charge separation taking place at the time when the intermediate defect configuration reaches the maximum barrier height along the reaction path. It is, however, very difficult to pin down the exact atomistic origin of the Fermi level influence on the barrier heights of the formation of charged vacancies, due to the lack of any theoretical support and the limitations of scanning tunneling microscopy, which does not allow to image directly the intermediate vacancy configurations during the vacancy formation process. The time resolution of the STM is in the order of $0.01 \mathrm{~s}$ or several orders of magnitude slower than required.

\section{CONCLUSIONS}

In conclusion, we investigated the kinetics of spontaneous thermal stoichiometry changes in (110) surfaces of III-V semiconductors arising from a selective vacancy formation. From the kinetic increase of the vacancy concentrations measured directly in scanning tunneling microscopy images we determined the barrier height for the formation of the positively charged As and $\mathrm{P}$ vacancies on GaAs and $\operatorname{InP}(110)$ surfaces. We found barrier heights in the range of 1.1-1.3 $\mathrm{eV}$. Furthermore, a quantitative analysis showed that the barrier height increases when the Fermi level at the surface moves toward midgap. This effect results in the observation of positively charged anion vacancies only on $p$-doped surfaces, but not on $n$-doped surfaces. An opposite direction of barrier height change is found for negatively charged $\mathrm{Ga}$ vacancies on $\mathrm{GaAs}(110)$, suggesting that only positively and negatively charged defects can be formed spontaneously on $p$ - and $n$-doped surfaces, respectively. Thus, the stoichiometry changes of $p$ - and $n$-doped GaAs(110) surfaces are in opposite direction, i.e., toward Ga-rich and As-rich surfaces, respectively. All presently available experiments therefore show that (110) surfaces of III-V semiconductors undergo spontaneous stoichiometry changes due to a selective vacancy formation, which is governed by the Fermi energy, which in turn is controlled by the vacancy concentration. Therefore we conclude that the surface Fermi-level controls the surface composition of (110) cleavage surfaces of III-V semiconductors. 


\section{ACKNOWLEDGMENTS}

The authors thank K. H. Graf for technical support and G. Schwarz for helpful discussions.

${ }^{1}$ R. M. Feenstra, J. M. Woodall, and G. D. Pettit, Phys. Rev. Lett. 71, 1176 (1993).

${ }^{2}$ C. Domke, Ph. Ebert, M. Heinrich, and K. Urban, Phys. Rev. B 54, 10288 (1996).

${ }^{3}$ Ph. Ebert, Surf. Sci. Rep. 33, 121 (1999).

${ }^{4}$ U. Semmler, Ph. Ebert, and K. Urban, Appl. Phys. Lett. 77, 61 (2000).

${ }^{5}$ M. B. Johnson, O. Albrektsen, R. M. Feenstra, and H. W. M. Salemink, Appl. Phys. Lett. 63, 2923 (1993); 64, 1454(E) (1994).

${ }^{6}$ J. F. Zheng, X. Liu, N. Newman et al., Phys. Rev. Lett. 72, 1490 (1994).

${ }^{7}$ J. F. Zheng, M. Salmeron, and E. R. Weber, Appl. Phys. Lett. 64, 1836 (1994); 65, 790(E) (1994).

${ }^{8}$ B. Siemens, C. Domke, M. Heinrich et al., Phys. Rev. B 59, 2995 (1999).

${ }^{9} \mathrm{Ph}$. Ebert, M. Heinrich, M. Simon et al., Phys. Rev. B 53, 4580 (1996).

${ }^{10}$ R. J. Hamers, J. Vac. Sci. Technol. B 6, 1462 (1988).

${ }^{11}$ J. A. Stroscio, R. M. Feenstra, and A. P. Fein, Phys. Rev. Lett. 58, 1668 (1987).

${ }^{12}$ C. Domke, M. Heinrich, Ph. Ebert, and K. Urban, J. Vac. Sci. Technol. B 16, 2825 (1998).

${ }^{13}$ G. Lengel, R. Wilkins, G. Brown, and M. Weimer, J. Vac. Sci. Technol. B 11, 1472 (1993).

${ }^{14} \mathrm{Ph}$. Ebert and K. Urban, Ultramicroscopy 49, 344 (1993).

${ }^{15}$ G. Lengel, R. Wilkins, G. Brown et al., Phys. Rev. Lett. 72, 836 (1994).

${ }^{16} \mathrm{Ph}$. Ebert, K. Urban, and M. G. Lagally, Phys. Rev. Lett. 72, 840 (1994).

${ }^{17}$ S. B. Zhang and A. Zunger, Phys. Rev. Lett. 77, 119 (1996).

${ }^{18}$ H. Kim and J. R. Chelikowsky, Phys. Rev. Lett. 77, 1063 (1996).

${ }^{19} \mathrm{Ph}$. Ebert, K. Urban, L. Aballe et al., Phys. Rev. Lett. 84, 5816 (2000).
${ }^{20}$ K.-J. Chao, A. R. Smith, and C. K. Shih, Phys. Rev. B 53, 6935 (1996).

${ }^{21} \mathrm{Ph}$. Ebert, X. Chen, M. Heinrich et al., Phys. Rev. Lett. 76, 2089 (1996).

${ }^{22} \mathrm{Ph}$. Ebert, M. Heinrich, M. Simon et al., Phys. Rev. B 51, 9696 (1995).

${ }^{23}$ M. Heinrich, Ph. Ebert, M. Simon et al., J. Vac. Sci. Technol. A 13, 1714 (1995).

${ }^{24} \mathrm{Ph}$. Ebert, T. Zhang, F. Kluge et al., Phys. Rev. Lett. 83, 757 (1999).

${ }^{25} \mathrm{G}$. Schwarz (private communication, 2000).

${ }^{26}$ J. Philibert, Atom Movements, Diffusion, and Mass Transport in Solids (Les Editions de Physique, Les Ulis, 1991).

${ }^{27}$ R. B. Dingle, Philos. Mag. 46, 861 (1955).

${ }^{28}$ Landolt-Börnstein, Zahlenwerte und Funktionen aus Naturwissenschaften und Technik (Springer, Berlin, 1982), Neue Serie Vol. III/17a.

${ }^{29}$ J. C. Brice, in Properties of Indium Phosphide (INSPEC, 1991), p. 18.

${ }^{30} \mathrm{H}$. Nienhaus and W. Mönch, Phys. Rev. B 50, 11750 (1994).

${ }^{31}$ H. Nienhaus and W. Mönch, Surf. Sci. Lett. 328, L561 (1995).

${ }^{32}$ W. G. Schmidt, F. Bechstedt, and G. P. Srivastava, Phys. Rev. B 52, 2001 (1995).

${ }^{33}$ J. Fritsch, P. Pavone, and U. Schröder, Phys. Rev. B 52, 11326 (1995).

${ }^{34}$ H. M. Tütüncü and G. P. Srivastava, Phys. Rev. B 53, 15675 (1996).

${ }^{35}$ U. Schröder, Physica B 219-220, 434 (1996).

${ }^{36}$ C. Eckl, J. Fritsch, P. Pavone, and U. Schröder, Surf. Sci. 394, 47 (1997).

${ }^{37}$ K. Hinrichs, A. Schierhorn, P. Haier et al., Phys. Rev. Lett. 79, 1094 (1997).

${ }^{38}$ R. J. Pechman, X.-S. Wang, and J. H. Weaver, Phys. Rev. B 51, 10929 (1995).

${ }^{39}$ G. Lengel, M. Wiemer, J. Gryko, and R. E. Allen, J. Vac. Sci. Technol. A 12, 1855 (1994)

${ }^{40}$ W. Mönch, Semiconductor Surfaces and Interfaces, Springer Series in Surface Science, edited by G. Ertl, R. Gomer, and D. L. Mills (SpringerVerlag, Berlin, 1993), Vol. 26. 